\title{
Bilateral Iliopsoas Hematomas Complicating Anticoagulant Therapy
}

\author{
Yuko WadA, Chie YanAgIHARA and Yo NishimURA
}

\begin{abstract}
An 85-year-old woman receiving anticoagulant therapy for transient ischemic attack suddenly developed bilateral femoral nerve palsy and severe pain in the bilateral groin and thighs. Her platelet count, prothrombin time and activated partial thromboplastin time were within the therapeutic range. Hematomas in the bilateral iliopsoas muscles were clearly detectable on CT scan. The right hematoma was larger than the left one and caused more severe femoral neuropathy, but improved gradually without surgical decompression. This case is reported here because bilateralism is exceptional, and iliopsoas hematoma should be suspected when a patient receiving anticoagulant therapy presents with pain in the groin or thigh.
\end{abstract}

(Internal Medicine 44: 641-643, 2005)

Key words: iliopsoas hematoma, embolization, anticoagulants

\section{Introduction}

The occurrence of iliopsoas hematoma during anticoagulant treatment is rare but well documented. Heparin and warfarin are the drugs involved in most of the reported cases, and iliopsoas hematoma in patients on anticoagulant treatment is usually unilateral (1). Although rare, bilateral hematoma may also occur.

Here, we report here a case of bilateral iliopsoas hematoma accompanied by massive bleeding and anemia during anticoagulant therapy.

\section{Case Report}

An 85-year-old woman was admitted for transient cerebral ischemia. She had a previous history of hypertrophic cardiomyopathy, but no history of bleeding diathesis.

On admission, her heart rate was sinus rhythm and blood pressure was 110/60. Ultrasound echocardiography showed that the lateral wall thickness of the left ventricle was 14 $\mathrm{mm}$, left ventricular (LV) motion was reduced (LVEF 44\%) and IVS (ed) was 1.0 (normal $0.5-1.3 \mathrm{~cm}$ ). LV inflow was pseudonormalized, which indicates reduction of the diastolic function. The Holter electrocardiogram showed no atrial fibrillation. During hospitalization, her systolic blood pressure increased from $110 \mathrm{mmHg}$ to $130 \mathrm{mmHg}$. Anticoagulant therapy was begun with 600 units of heparin infusion per hour, and the dose was adjusted to maintain the activated partial thromboplastin time (APTT) at 50-60 seconds. Warfarin sodium was added at a starting dose of $3 \mathrm{mg}$ daily.

After 3 days of heparin and warfarin treatment, the patient complained of a sudden onset of severe pain in the right groin and the anterior aspect of the right thigh, which forced her to keep her right hip flexed. Because of the pain, the degree of muscle weakness was difficult to ascertain, but the patient showed reduced sensation to pinpricks in the distribution area of the right saphenous nerve. The patient was afebrile without any swelling or change in skin color. The next day she developed similar but less intense symptoms on the left side. The neurological examination revealed bilateral femoral nerve palsy, more severe on the right than on the left side. The femoral and foot pulses were normal. An enhanced computed tomography (CT) scan of the abdomen and pelvis demonstrated the presence of bilateral iliopsoas hematomas, with the right hematoma larger than the left, while seeping of the contrast material into the hematomas indicated active bleeding (Fig. 1). When the symptoms started, the patient's prothrombin time international normalized ratio (PT-INR) was 1.5 , activated partial thromboplastin time (APTT) was 56.8 seconds (normal: $25-40$ seconds), her hemoglobin was $10.7 \mathrm{~g} / \mathrm{l}(10.8 \mathrm{~g} / \mathrm{l}$ on admission) and the platelet count was $15.3 \times 10^{5} / l\left(15.5 \times 10^{5} / l\right.$ on admission $)$. Warfarin was discontinued immediately and heparin was reduced, but her condition gradually deteriorated during the four days after onset of the symptoms.

From Department of Neurology, Nishi-Kobe Medical Center, Kobe

Received for publication August 27, 2004; Accepted for publication January 5, 2005

Reprint requests should be addressed to Dr. Yuko Wada, Department of Neurology, Nishi-Kobe Medical Center, 5-7-1 Kouji-Dai, Nishi-ku, Kobe 651-2273 


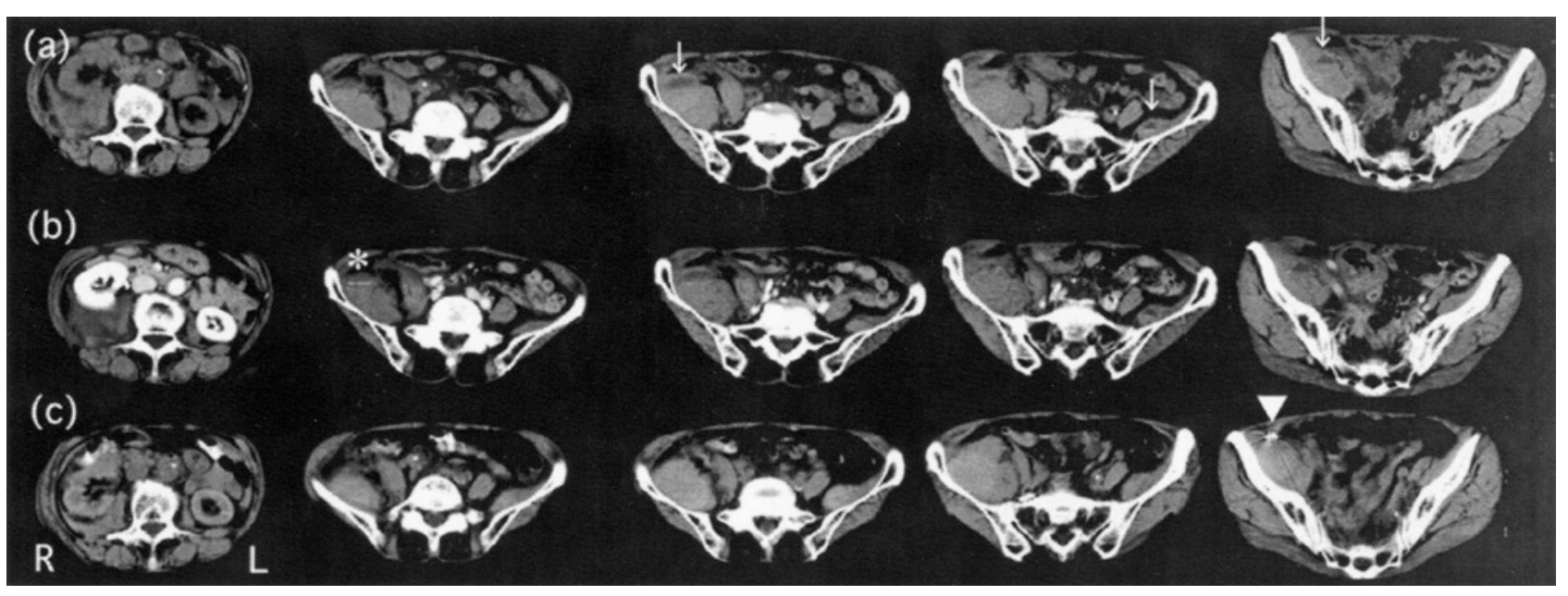

Figure 1. Enhanced computed tomography (CT) scan of the pelvis. a) Plain CT scan. b) Enhanced CT scan. c) Plain CT scan after TAE. Low-attenuation areas are seen in both iliopsoas muscles (arrows). Note the extravasation of contrast material from the right hematoma indicating active bleeding (asterisk). Arrowhead: coil.

Table 1. Cases of Bilateral Iliopsoas Hematomas Associated with Anticoagulant Therapy Reported in the Literature

\begin{tabular}{|c|c|c|c|c|c|c|}
\hline Author (year) & Age/Sex & $\begin{array}{l}\text { Indication for } \\
\text { anticoagulation }\end{array}$ & $\begin{array}{c}\text { Type of } \\
\text { anticoagulant }\end{array}$ & $\begin{array}{c}\text { Onset after } \\
\text { administration } \\
\text { of anticoagulant }\end{array}$ & Coagulation level & Treatment \\
\hline Storen (1978) & 55 y.o, F & DVT & heparin/warfarin & 5 days & therapeutic & surgery \\
\hline Barontini (1986) & 65 у.о, F & MI & $\begin{array}{l}\text { antiaggregant } \\
\quad(\text { Teklid } 2 \mathrm{cps}) \\
\text { anticoagulant } \\
\quad(\text { Calcipatina } 0.5 \times 2)\end{array}$ & $\begin{array}{l}9 \text { months } \\
3 \text { months }\end{array}$ & $\begin{array}{l}\text { normal (prothrombin } \\
\text { activity PTT, fibrino- } \\
\text { gen, platelets) }\end{array}$ & conservatively \\
\hline Niakan (1991) & 54 у.о, F & $\mathrm{PE}$ & heparin/warfarin & NM & $\mathrm{NM}$ & $\begin{array}{l}\text { rt side surgery } \\
\text { It side conserva- } \\
\text { tively }\end{array}$ \\
\hline Jamjoom (1993) & 19 у.о, F & DVT & heparin/warfarin & 3 weeks & therapeutic & surgery \\
\hline Our case & 85 у.о, F & TIA & heparin/warfarin & 3 days & therapeutic & $\begin{array}{l}\text { conservatively } \\
\text { (plus TAE?) }\end{array}$ \\
\hline
\end{tabular}

DVT: deep venous thrombosis; MI: myocardial infarct; PE: pulmonary embolism; TIA: transient ischemic attack; NM: not mentioned

By the fourth day, the platelet count was $13.8 \times 10^{5} / l$, PTINR was 1.8 , APTT was 40.8 seconds, and hemoglobin had dropped to a minimum of $7.3 \mathrm{~g} / \mathrm{l}$, so that a blood transfusion was administered. Since the patient was judged to be a poor candidate for surgery because of her heart function, transcatheter arterial embolization (TAE) of the right side was judged to be the treatment of choice. Aortography via the right femoral artery approach using a 5-Fr catheter was performed to determine the site of bleeding, but no contrast extravasation was observed. Although bleeding might have improved spontaneously during angiography, we decided to perform TAE to prevent rebleeding. Embolization with a resorbable gelatinous sponge fragment was performed at the level of the third and fourth right lumbar artery trunk, because bleeding in iliopsoas hematoma usually originates from the third or fourth lumbar artery. Embolization with both the resorbable gelatinous sponge fragment and a micro coil occlusion device was also performed at the level of the right deep circumflex artery and right iliolumbar artery. Two days after TAE, CT findings showed that the hematomas had not increased in size and the low-attenuation areas had disappeared (Fig. 1c). The patient improved progressively and gradual pain reduction and recovery of strength in her legs were noted over the next few days. On discharge three weeks after TAE, the femoral nerve function had returned to normal apart from a mild residual weakness in the right quadriceps muscle. 


\section{Bilateral Iliopsoas Hematomas}

\section{Discussion}

The present patient suffered bilateral iliopsoas hematomas while undergoing anticoagulant therapy. Hemorrhage into the iliacus, psoas and iliopsoas muscles is an infrequent complication of anticoagulant therapy and is usually unilateral (1). To the best of our knowledge, however, bilateral hematomas have rarely been reported (2-5) (Table 1). In our case, daily examination of the patient's blood indicated that APTT and PT-INR were within the therapeutic range when the bleeding started, and before occurrence of the initial symptoms. In other cases of bilateral hematomas (Table 1), APTT and PT-INR of most patients were in the therapeutic anticoagulation range at the onset of bleeding symptoms. Sasson et al (1) reported that in the majority of cases of unilateral hematoma these values were also in the therapeutic anticoagulation range.

Patients with iliopsoas hematoma manifest a wide range of symptoms, from early signs, such as lumbar or groin pain, to lumbar plexus neuropathy or massive bleeding and shock. It is a matter of controversy whether such hematomas should be surgically evacuated or conservatively treated (6). Conservative management is sufficient for cases with small hematomas and mild symptoms of femoral neuropathy, while some authors have recommended immediate surgical decompression in cases of life-threatening complications of iliopsoas hemorrhage. However, surgical management constituted a risk factor for the present patient because of her heart dysfunction, and there are no established guidelines for the management of such patients. Recently, the first successful treatment using TAE for severe psoas hematoma secondary to anticoagulant therapy was reported by Qanadli et al
(6). On the basis of this success with treatment using TAE, we decided to use TAE to stop the active bleeding even though active bleeding in our patient might have improved spontaneously during angiography. In view of our result, we would advocate that, because of its lower morbidity, TAE should be considered rather than surgery.

In summary, the possibility of iliopsoas hematoma should be considered in any patient undergoing anticoagulant treatment, even if APTT and PT-INR values are in the therapeutic' anticoagulation range. In the presence of severe anemia or neurological deficit, enhanced CT examination of the pelvis must be performed immediately to detect any active bleeding in the iliopsoas muscle. If such bleeding is found, TAE might be a safer therapeutic approach than surgery in addition to other significant advantages.

\section{References}

1) Sasson Z, Mangat I, Peckham KA. Spontaneous iliopsoas hematoma in patients with unstable coronary syndromes receiving intravenous heparin in therapeutic doses. Can J Cardiol 12: 490-494, 1996.

2) Storen EJ. Bilateral iliacus haematoma with femoral nerve palsy complicating anticoagulant therapy. Acta Chir Scand 144: 181-183, 1978.

3) Barontini F, Macucci M. Simultaneous femoral nerve palsy due to hemorrhage in both iliac muscles. Ital J Neurol Sci 7: 463-465, 1986.

4) Niakan E, Carbone JE, Adams M, Schroeder F. Anticoagulants, iliopsoas haematoma and femoral nerve compression. Am Fam Physician 44: 2100-2102, 1991.

5) Jamjoom ZA, al-Bakry A, al-Momen A, Malabary T, Tahan AR, Yacub B. Bilateral femoral nerve compression by iliacus hematomas complicating anticoagulant therapy. Surg Today 23: 535-540, 1993.

6) Qanadli SD, EI Hajjam, Mignon F, Bruckert F, Chagnon S, Lacombe P. Life-threatening spontaneous psoas haematoma treated by transcatheter arterial embolization. Eur Radiol 9: 1231-1234, 1999. 